\title{
Extracting edge radiation within a straight section of an electron storage ring
}

\author{
R. A. Bosch \\ Synchrotron Radiation Center, University of Wisconsin-Madison, 3731 Schneider Drive, Stoughton, Wisconsin 53589
}

(Received 10 December 2001; published 19 February 2002)

\begin{abstract}
Edge radiation may be extracted within a straight section of an electron storage ring by using a tilted mirror with a hole (or slot) for passage of the electron beam and its velocity field. At wavelengths much greater than the critical wavelength of the ring's synchrotron radiation, the flux distribution upon the mirror is almost independent of wavelength. When the reflected radiation is extracted through a nearby window, diffraction reduces the extracted flux at wavelengths exceeding $\sim 2 \pi$ times the hole radius. For the Aladdin $800 \mathrm{MeV}$ electron storage ring, the extracted edge radiation may find application as a broadband source for wavelengths of $1 \mu \mathrm{m}-60 \mathrm{~mm}$ or as a diagnostic of the bunch dimensions.
\end{abstract}

DOI: 10.1103/PhysRevSTAB.5.020701

PACS numbers: 41.60.Ap, 07.57.Hm

\section{INTRODUCTION}

Long-wavelength radiation may be extracted within a straight section of an ultrarelativistic electron storage ring by using a tilted mirror with a hole (or slot) for passage of the electron beam [1-4]. When the hole subtends a half-angle exceeding $1 / \gamma$ with respect to the entrance of the straight section (where $\gamma$ is the relativistic factor), the mirror intercepts little of the Liénard-Wiechart velocity field produced within the straight section [5]. In this case, the extracted radiation at wavelengths much greater than the critical wavelength is primarily edge radiation [5-8], which is emitted at the bending magnet edge upstream of the straight section.

At these long wavelengths, the calculated flux distribution upon the mirror is nearly independent of wavelength [5-8], consistent with measurements at wavelengths up to $200 \mu \mathrm{m}[6,7]$. A long-wavelength cutoff of the extracted radiation is expected when the edge radiation is not efficiently reflected. We previously estimated that this occurs for wavelengths exceeding $\sim 2 \pi$ times the radius of the hole (or $\sim 2 \pi$ times the half-gap of a slotted mirror) [5]. For the Aladdin electron storage ring, wavelengths exceeding $\sim 60 \mathrm{~mm}$ are expected to be cut off with a hole radius or half gap of $9.5 \mathrm{~mm}$ [5]. In contrast, a feasible large aperture for ordinary synchrotron radiation or edge radiation downstream of an Aladdin straight section is computed to cut off wavelengths exceeding $\sim 500 \mu \mathrm{m}$ [9].

Because the main apparent advantage of extraction within a straight section is the increased cutoff wavelength, it is important to verify its value by computation of the extracted radiation. In this article, the extracted radiation is computed by evaluating the Kirchoff scalar diffraction integral [10] for each component of the radiation field. We demonstrate the validity of this method by comparison with previous results, and obtain an approximate formula for the extracted flux. For Aladdin, we compute the spatial distribution of extracted radiation for a mirror with no hole that extracts edge and diffraction radiation and for a mirror with a hole that extracts edge radiation. When radiation is extracted through a window in close proximity to the mirror, the flux is cut off at wavelengths exceeding $\sim 60 \mathrm{~mm}$ in both cases.

However, a mirror without a hole is not feasible at Aladdin, where a long beam lifetime is required. For a ring current of $200 \mathrm{~mA}$, a mirror with a hole of 9.5-mm radius is expected to extract an incoherent flux of $6.8 \times 10^{12}$ photons $/ \mathrm{s}-0.1 \%$ bandwidth for wavelengths of $1 \mu \mathrm{m}-60 \mathrm{~mm}$. The extracted edge radiation may be useful as a diagnostic of the bunch dimensions or as a broadband infrared and microwave source.

\section{INFINITE-LENGTH STRAIGHT SECTION}

\section{A. Backward diffraction and transition radiation}

When an ultrarelativistic electron travels in a straight line for all time, a mirror that intercepts its Coulomb field extracts radiation. Consider the radiation reflected in the backward direction by a perfectly conducting mirror lying in a plane perpendicular to the electron's path. Let the electron's path be the $z$ axis with $z=0$ at the mirror and time $t=0$ when the electron passes the mirror. We use spherical coordinates $\left(r, \theta_{1}=\pi / 2, \phi_{1}\right)$ to describe the $z=0$ plane, while $(R, \theta, \phi)$ describe an observer of the reflected radiation, with $\theta=0$ in the backward direction.

The radiation field incident upon the mirror is given by the Lorentz transform of the Coulomb field. For $\gamma \gg 1$, nearly all of the field is radially polarized $[\vec{E}=E(r) \hat{r}]$, with radial component at wavelength $\lambda$ in SI units [10],

$$
E_{\mathrm{Coul}}(r)=\int_{-\infty}^{\infty} E(r, t) e^{i \omega t} d t=\left(\frac{e}{\varepsilon_{0} c \lambda \gamma}\right) K_{1}\left(\frac{2 \pi r}{\lambda \gamma}\right) .
$$

Here, $\omega$ is angular frequency, $c$ is the speed of light, $e<0$ is the electron charge, $\varepsilon_{0}$ is the permittivity of free space, and $K_{1}$ is a modified Bessel function of the second kind. $E_{\text {Coul }}$ is the Liénard-Wiechart velocity field of an electron that travels at constant velocity for all time [10], and is 
proportional to $1 / r$ for $r \ll \lambda \gamma / 2 \pi$ and nearly zero for $r>\lambda \gamma$.

According to Kirchoff scalar diffraction theory, each component of the reflected radiation at an observer location with $R \gg \lambda / 2 \pi$ and $\theta \ll 1$ is given by a scalar diffraction integral with obliquity factor equaling one, so that [10]

$$
\vec{E}(R, \theta, \phi)=\frac{-i}{\lambda} \int d A \vec{E}\left(r, \pi / 2, \phi_{1}\right) \exp (i k D) / D .
$$

The integration is over the mirror, where $\vec{E}$ is the reflected field, $k=2 \pi / \lambda$, and $d A=r d r d \phi_{1}$. Here, $D$ is the distance between $\left(r, \theta_{1}=\pi / 2, \phi_{1}\right)$ and $(R, \theta, \phi)$, obeying for $r \ll R$

$$
D \approx R+r^{2} / 2 R-r \sin \theta\left(\cos \phi_{1} \cos \phi+\sin \phi_{1} \sin \phi\right),
$$

where $\sin (\theta) \approx \theta$ for $\theta \ll 1$. When $\vec{E}$ is radially polarized and the mirror is an annulus of radius $b$ containing a hole of radius $a$, it is sufficient to consider an observer with $\phi=0$. Integrating over $\phi_{1}[11]$ yields the radial component of the observer's field,

$$
\begin{aligned}
E(R, \theta)= & -e^{i k R}\left(\frac{2 \pi}{R \lambda}\right) \int_{a}^{b} r d r E(r) J_{1}(k r \theta) \\
& \times \exp \left(\frac{i k r^{2}}{2 R}\right)
\end{aligned}
$$

where $E(r)$ is the radial component of the reflected field on the mirror. Letting $E(r)=-E_{\text {Coul }}(r)$ gives the Kirchoff diffraction integral for backward diffraction radiation,

$$
\begin{aligned}
E(R, \theta)= & e^{i k R}\left(\frac{2 \pi e}{R \gamma \lambda^{2} \varepsilon_{0} c}\right) \int_{a}^{b} r d r K_{1}\left(\frac{2 \pi r}{\lambda \gamma}\right) \\
& \times J_{1}(k r \theta) \exp \left(\frac{i k r^{2}}{2 R}\right) .
\end{aligned}
$$

When a mirror occupies the entire $z=0$ plane $(a=0$, $b=\infty)$, the radiation is called backward transition radiation. Because $K_{1}(x) \approx 0$ for $x \gg 1$, the integrand of Eq. (5) is nearly zero for $r>\lambda \gamma$. In the far field where $R \gg \lambda \gamma^{2}$, approximating $\exp \left(i k r^{2} / 2 R\right) \approx 1$ in Eq. (5) gives [12]

$$
E_{\mathrm{far}}(R, \theta)=e^{i k R} \frac{e \gamma^{2} \theta}{2 \pi R \varepsilon_{0} c\left(1+\gamma^{2} \theta^{2}\right)} .
$$

The flux distribution of far-field transition radiation is independent of wavelength, and peaks when $\theta=1 / \gamma$. In the near field where $R \ll \lambda \gamma^{2}$ and $R \theta \ll \lambda \gamma$, taking the $\gamma \rightarrow \infty$ limit where $K_{1}(x) \rightarrow 1 / x$ in Eq. (5) gives [13]

$$
E(R, \theta)=e^{i k R} \frac{i e}{\pi R \varepsilon_{0} c \theta} \sin \left(\frac{\pi R \theta^{2}}{2 \lambda}\right) \exp \left(\frac{-i \pi R \theta^{2}}{2 \lambda}\right) .
$$

The near field radiation peaks when $\theta \approx(\lambda / R)^{1 / 2}$. As expected by the method of images, the backward transition radiation given by the Kirchoff integral is the opposite of the radiation field from a suddenly deflected electron $[14,15]$.

For $R<\lambda / 2 \pi$, Eqs. (2)-(7) do not apply. Since transition radiation is equivalent (up to a sign) to the radiation from a suddenly deflected electron, the backward transition radiation primarily has a longitudinal polarization for $R \ll \lambda[14]$.

When the mirror occupies part of the $z=0$ plane, the radiation is known as backward diffraction radiation. Since $\int_{a}^{b}=\int_{a}^{\infty}-\int_{b}^{\infty}$, it is sufficient to evaluate Eq. (5) for $b=\infty$. In the far field where $R \gg \lambda \gamma^{2}$, approximating $\exp \left(i k r^{2} / 2 R\right) \approx 1$ in Eq. (5) gives, for $b=\infty$ [16],

$$
\begin{aligned}
E(R, \theta)= & e^{i k R} \frac{e \gamma^{2} \theta}{2 \pi R \varepsilon_{0} c\left(1+\gamma^{2} \theta^{2}\right)} \\
\times & {\left[\frac{2 \pi a}{\lambda \gamma} K_{1}\left(\frac{2 \pi a}{\lambda \gamma}\right) J_{0}\left(\frac{2 \pi a \theta}{\lambda}\right)\right.} \\
& \left.+\frac{2 \pi a}{\lambda \gamma^{2} \theta} K_{0}\left(\frac{2 \pi a}{\lambda \gamma}\right) J_{1}\left(\frac{2 \pi a \theta}{\lambda}\right)\right] .
\end{aligned}
$$

To extract a substantial amount of radiation requires that $a \ll \lambda \gamma / 2 \pi$, in which case Eq. (8) becomes $[1,3]$

$$
E(R, \theta) \approx e^{i k R} \frac{e \gamma^{2} \theta}{2 \pi R \varepsilon_{0} c\left(1+\gamma^{2} \theta^{2}\right)} J_{0}\left(\frac{2 \pi a \theta}{\lambda}\right) .
$$

Since $J_{0}(x) \approx 1$ for $x<1$, the far-field diffraction radiation through an aperture subtending $\left\{\theta<\theta_{\text {ap }}\right\}$ is independent of $a$ for $a<\lambda / 2 \pi \theta_{\mathrm{ap}}$, indicating that little flux is obtained from the mirror region where $r<\lambda / 2 \pi \theta_{\mathrm{ap}}$. Approximating the incoherent flux of far-field diffraction radiation into $\left\{\theta<\theta_{\text {ap }}\right\}$ as the flux incident upon the mirror region where $r>\lambda / 2 \pi \theta_{\text {ap }}$ [17], and approximating $K_{1}(x) \approx 1 / x$ for $x<1$ and 0 elsewhere, we obtain

$$
\text { flux }\left(\theta<\theta_{\text {ap }}\right) \approx 2\left[\alpha \frac{\Delta \omega}{\omega} \frac{I}{e \pi}\right] \ln \left[\frac{\min (b, \lambda \gamma / 2 \pi)}{\max \left(a, \lambda / 2 \pi \theta_{\text {ap }}\right)}\right],
$$

where $\alpha=1 / 137$ is the fine structure constant, $\Delta \omega / \omega$ is the relative bandwidth, and $I<0$ is the storage ring current. A flux of zero [i.e., $\ll \alpha(\Delta \omega / \omega)(I / e \pi)$ ] is expected when Eq. (10) yields a negative value.

\section{B. Forward diffraction and transition radiation}

Consider an observer downstream of the mirror. We refer to the portion of the $z=0$ plane that is not occupied by the mirror as the "opening," and the radiation from the opening as $E_{\text {open }}$. Radiation also arises from the portion of the electron orbit downstream of the mirror. The LiénardWiechart velocity field generated downstream of the mirror $\left(E_{v_{\text {exit }}}\right)$ by itself does not obey Maxwell's equations. A solution with the correct boundary condition may be obtained by summing $E_{v_{\text {exit }}}$ with the field of far-field transition radiation $E_{\text {far }}$. The field of forward diffraction radiation is therefore 


$$
E=E_{v_{\text {exit }}}+E_{\text {far }}+E_{\text {open }}
$$

$E_{v_{\text {exit }}}$ nearly equals $E_{\text {Coul }}$ for $\theta<1 / \gamma$ and nearly vanishes for $\theta>1 / \gamma$ [5]. Equation (11) may also be written as

$$
E=E_{\text {Coul }}+E_{\text {mirror }},
$$

where $E_{\text {mirror }}$ describes the backwards diffraction radiation from the mirror.

When $E_{\text {open }}$ is negligible (e.g., the mirror fills the entire $z=0$ plane), the radiation is called forward transition radiation, which obeys

$$
E=E_{v_{\text {exit }}}+E_{\text {far }}=E_{\text {Coul }}-E_{v_{\text {entrance }}}+E_{\text {far }},
$$

where $E_{v_{\text {entrance }}}$ is the portion of the Coulomb field produced when $z<0[5,14]$. For an observer with $\theta<1 / \gamma$, $E \approx E_{\text {Coul }}$, while for $\theta>1 / \gamma, E \approx E_{\text {far }}$ [5]. For $\theta>$ $1 / \gamma$, the radiation distribution is nearly independent of wavelength.

The radiation within a finite-length straight section of an electron storage ring is equivalent to forward transition radiation at wavelengths where the edge radiation equals that from a sudden deflection [5]. This applies for wavelengths $\lambda \gg d / \gamma^{2}$, where $d$ is the distance required to deflect an electron through an angle of $1 / \gamma$ at the bending magnet edge. For a hard-edged bending magnet, this criterion is obeyed for wavelengths long compared to the critical wavelength of the bending magnet's synchrotron radiation. For Aladdin, the radiation from a sudden deflection provides a good approximation for $\lambda>1 \mu \mathrm{m}[5,14]$.

\section{FINITE STRAIGHT SECTION LENGTH}

Consider the radiation reflected in the backward direction by a mirror at a distance $L$ from the upstream end of a straight section for $\lambda \gg d / \gamma^{2}$, or at a distance $L$ from a source of forward transition radiation. We let $z=0$ at the mirror position and $t=0$ when an electron passes the mirror. The Kirchoff diffraction integral is given by Eq. (2), where the reflected field $\vec{E}$ is the negative of the incident field given by Eq. (13). For an annular mirror, Eq. (4) gives the backward radiation for an observer at $(R, \theta, \phi)$ as

$$
E(R, \theta)=e^{i k R}\left(\frac{2 \pi}{R \lambda}\right) \int_{a}^{b} r d r\left[E_{\mathrm{Coul}}(r)+E_{\mathrm{far}}(r)-E_{v_{\text {entrance }}}(r)\right] J_{1}(k r \theta) \exp \left(\frac{i k r^{2}}{2 R}\right) .
$$

In the integrand of Eq. (14), the fields should be evaluated in a coordinate system where $t=0$ when the electron passes through the mirror. In this coordinate system, $E_{\text {Coul }}(r)$ is given by Eq. (1). Letting $\theta=r / L$ and $R=\left(L^{2}+r^{2}\right)^{1 / 2} \approx$ $L+r^{2} / 2 L$ in Eq. (6), and multiplying by $\exp (-i \omega L / \beta c)$ to account for the difference in time coordinate gives

$$
E_{\mathrm{far}}(r)=\frac{e \gamma^{2}(r / L)}{2 \pi L \varepsilon_{0} c\left(1+\gamma^{2} r^{2} / L^{2}\right)} \exp \left(-i k L / 2 \gamma^{2}+i k r^{2} / 2 L\right)
$$

while in the coordinate system employed here [14]

$$
E_{v_{\text {entrance }}}(r)=\frac{e}{\pi \varepsilon_{0} c r} \int_{0}^{\gamma r / L} \frac{w}{\left(1+w^{2}\right)^{2}} \exp \left[\frac{i \pi r}{\lambda \gamma}\left(w-\frac{1}{w}\right)\right] d w .
$$

Equations (1) and (14)-(16) constitute a solution for cylindrically symmetric backwards radiation when $\theta \ll 1$ and $R \gg \lambda / 2 \pi$.

Consider a mirror that fills the entire $z=0$ plane. For $L>\lambda \gamma^{2}, E_{v_{\text {entrance }}}$ is negligible [14], so the backward radiation is the superposition of a transition radiation source located at the mirror and an edge radiation source located a distance of $L$ upstream, equivalent to the superposition of two edge radiation sources. This agrees with the method of images.

When $L \ll \lambda \gamma^{2}$, the radiation incident upon the mirror approximately equals $E_{\text {Coul }}(r)$ for $r<L / \gamma$, where we may approximate $K_{1}(x) \approx 1 / x$ and multiply by $\exp \left(i k r^{2} / 2 L\right) \approx 1$. For $r>L / \gamma$, the incident radiation approximately equals $E_{\text {far }}(r)$, where we may approximate $\left(1+\gamma^{2} r^{2} / L^{2}\right) \approx \gamma^{2} r^{2} / L^{2}$ and $\exp \left(-i k L / 2 \gamma^{2}\right) \approx 1$. Thus, the incident radiation field approximately equals $\left(e / 2 \pi \epsilon_{0} c r\right) \exp \left(i k r^{2} / 2 L\right)$ for all $r$, so that Eq. (14) integrates to [13]

$$
\begin{aligned}
E(R, \theta)= & e^{i k R} \frac{i e}{\pi R \varepsilon_{0} c \theta} \sin \left(\frac{\pi R_{\mathrm{eff}} \theta^{2}}{2 \lambda}\right) \\
& \times \exp \left(\frac{-i \pi R_{\mathrm{eff}} \theta^{2}}{2 \lambda}\right),
\end{aligned}
$$

where $R_{\text {eff }} \equiv R L /(R+L)$. The incoherent flux into $\left\{\theta<\theta_{\text {ap }}\right\}$ equals [15]

$$
\begin{aligned}
\operatorname{flux}\left(\theta<\theta_{\mathrm{ap}}\right)=2 \alpha \frac{\Delta \omega}{\omega} \frac{I}{e \pi} & {\left[C+\ln \left(\frac{\pi R_{\mathrm{eff}} \theta_{\mathrm{ap}}^{2}}{\lambda}\right)\right.} \\
& \left.-\operatorname{ci}\left(\frac{\pi R_{\mathrm{eff}} \theta_{\mathrm{ap}}^{2}}{\lambda}\right)\right],
\end{aligned}
$$

where $C=0.577215 \ldots$ is Euler's constant and ci is the cosine integral (also written $\mathrm{Ci}$ ). An aperture that subtends $\left\{\theta<\theta_{\text {ap }}\right\}$ cuts off wavelengths exceeding $\sim R_{\text {eff }} \theta_{\text {ap }}^{2}$.

As expected by the method of images, Eq. (17) is the opposite of an electron's edge radiation at a distance $R$ 
downstream of a straight section of length $L \ll \lambda \gamma^{2}$, when either $R \ll \lambda \gamma^{2}$ or $R \gg \lambda \gamma^{2}$ is obeyed [15,18]. Equation (17) also agrees with numerical computations [19] and experimental results [20] for edge radiation. The validity of Eq. (17), obtained from Eq. (14) when $a=0$ and $b=\infty$, supports the validity of Eq. (14) for arbitrary mirror dimensions.

When a mirror subtends a half-angle $<1 / \gamma$ with respect to the upstream end of the straight section $(b / L<1 / \gamma)$, the radiation field upon the mirror is nearly unaffected by the finite length of the straight section and approximately equals $E_{\text {Coul }}$. The extracted radiation, known as backward diffraction radiation [1-4], may be evaluated using Eqs. (5) and (8)-(10). Equations (6) and (7) also apply when $b>\lambda \gamma$ and $a=0$.
When the hole in a mirror subtends a half-angle $>1 / \gamma$ with respect to the upstream end of the straight section $(a / L>1 / \gamma)$, the field upon the mirror is primarily edge radiation, whose flux distribution is independent of wavelength. As in the case of backward diffraction radiation, the radiation field upon the portion of the mirror where $r<\lambda / 2 \pi \theta_{\text {ap }}$ varies on a scale length $<\lambda / 2 \pi \theta_{\text {ap }}$, and is therefore expected to diffract by an angle exceeding $\theta_{\text {ap. }}$ Thus, when edge radiation is extracted through an aperture subtending $\left\{\theta<\theta_{\text {ap }}\right\}$ that is sufficiently large to collect a specular reflection, the extracted flux is approximately given by that incident upon the mirror region where $r>\lambda / 2 \pi \theta_{\mathrm{ap}}$. The incoherent flux of extracted edge radiation may therefore be approximated by $[14,15]$

$$
\text { flux }\left(\theta<\theta_{\text {ap }}\right) \approx \alpha \frac{\Delta \omega}{\omega} \frac{I}{e \pi}\left\{\left[\ln \left(1+\gamma^{2} \theta_{\text {mirror }}^{2}\right)-\frac{\gamma^{2} \theta_{\text {mirror }}^{2}}{1+\gamma^{2} \theta_{\text {mirror }}^{2}}\right]-\left[\ln \left(1+\gamma^{2} \theta_{\text {min }}^{2}\right)-\frac{\gamma^{2} \theta_{\text {min }}^{2}}{1+\gamma^{2} \theta_{\text {min }}^{2}}\right]\right\},
$$

where $\quad \theta_{\text {mirror }} \equiv b / L, \quad \theta_{\min } \equiv \max \left(a / L, \lambda / 2 \pi \theta_{\text {ap }} L\right)$. When Eq. (19) yields a negative value, a flux of zero [i.e., $\ll a(\Delta \omega / \omega)(I / e \pi)]$ is expected. Because of diffraction, a reduction in the extracted flux is expected above a cutoff wavelength of $2 \pi a \theta_{\text {ap }}$. When a large aperture with $\theta_{\text {ap }} \approx 1$ is used to extract most of the reflected radiation, the expected cutoff wavelength is $\sim 2 \pi$ times the radius of the hole.

\section{EDGE RADIATION EXTRACTION AT ALADDIN}

At the Aladdin $800 \mathrm{MeV}$ electron storage ring [21], radiation may be extracted within a straight section by a $45^{\circ}$ mirror filling the cylindrical vacuum chamber, whose radius is $32 \mathrm{~mm}$. The radiation then passes through a circular window of 32-mm radius at a distance $R_{0}$ from the straight section axis, as shown in Fig. 1. $R_{0}=32 \mathrm{~mm}$ describes a window flush with the vacuum chamber wall, while $R_{0}=$ $64 \mathrm{~mm}$ describes a window mounted in a standard flange. For comparison, we also consider $R_{0}$ equaling 128 or

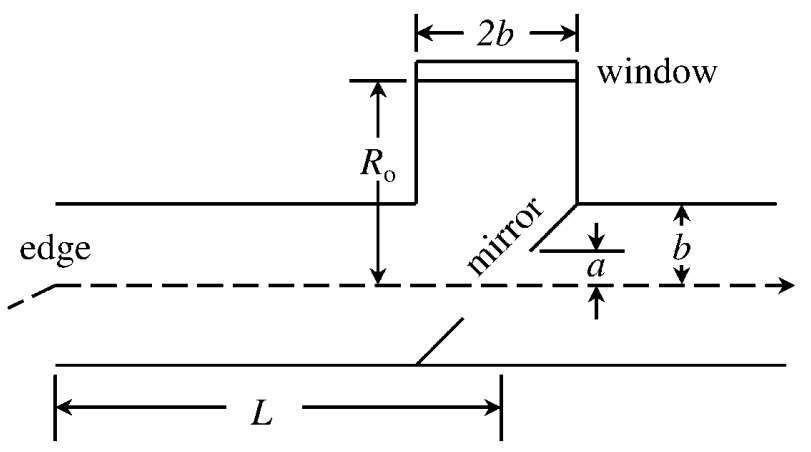

FIG. 1. Geometry for extraction of edge radiation within an Aladdin straight section, where $L=3 \mathrm{~m}, a=9.5 \mathrm{~mm}$, and $b=32 \mathrm{~mm}$. Values of $R_{0}$ that are considered are $32,64,128$, and $256 \mathrm{~mm}$.
$256 \mathrm{~mm}$. It is possible to extract radiation at a distance $L$ of $3 \mathrm{~m}$ from the straight section entrance, in which case the mirror subtends a half-angle of $16.71 / \gamma$ with respect to the straight section entrance. Since $R_{0} \ll L$, the window will collect nearly all of the specularly reflected edge radiation.

For $\gamma \gg 1$, the extracted radiation is expected to depend only slightly upon the tilt angle of the mirror [3], so we
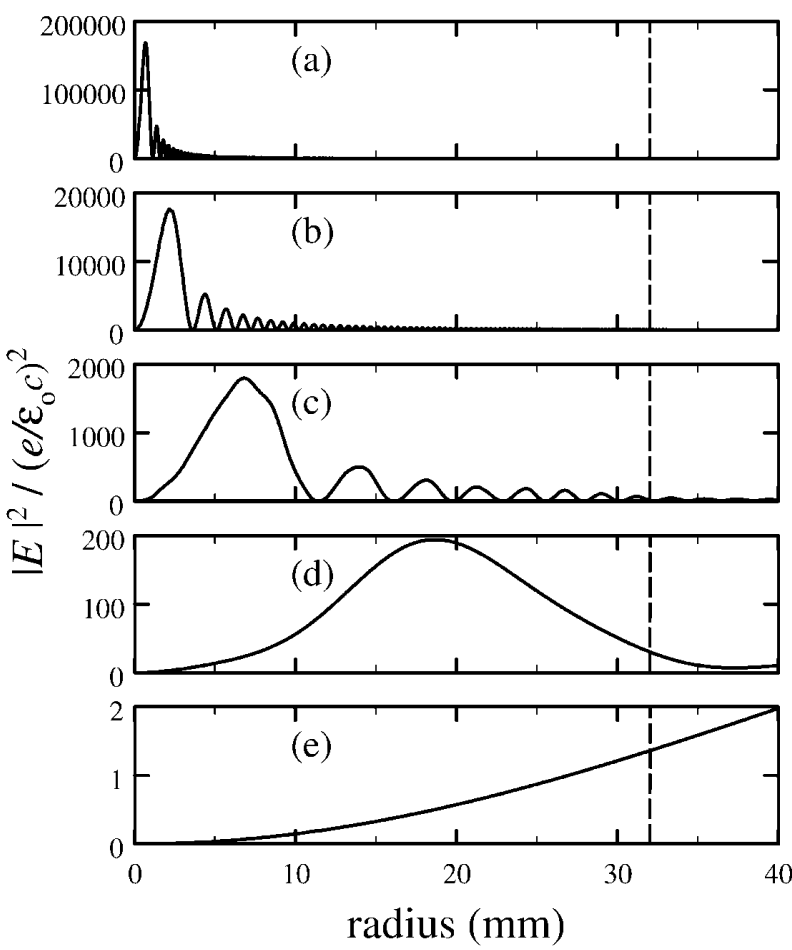

FIG. 2. Radiation distribution at the extraction window from a mirror with no hole when the window is located $64 \mathrm{~mm}$ from the straight section axis. The dashed line shows the $32-\mathrm{mm}$ radius of the window. (a) $\lambda=10 \mu \mathrm{m}$, (b) $\lambda=100 \mu \mathrm{m}$, (c) $\lambda=1 \mathrm{~mm}$, (d) $\lambda=10 \mathrm{~mm}$, and (e) $\lambda=100 \mathrm{~mm}$. 
model cylindrically symmetric backward extraction, with $b=32 \mathrm{~mm}$. We first consider a thin-foil mirror with no hole $(a=0)$, which extracts edge radiation and diffraction radiation. Figure 2 shows the radiation from an electron for a window mounted in a standard flange $\left(R_{0}=64 \mathrm{~mm}\right)$, computed from Eq. (14). For observer locations within the window, the radiation distribution nearly equals that of a mirror with infinite extent [given by Eq. (17)] for $10 \mu \mathrm{m} \leq \lambda \leq 10 \mathrm{~mm}$. The finite mirror size has little effect because the mirror is as large as the window. At much longer wavelengths, little radiation is extracted. Equations (14) and (17) become inaccurate at wavelengths exceeding $\sim 2 \pi R=400 \mathrm{~mm}$, where longitudinally polarized radiation is expected to predominate [14].

Figure 3 shows the computed incoherent flux through the window and that given by Eq. (18) for a mirror of infinite extent. The close agreement confirms that the finite mirror size has little effect on the extracted flux. The flux decreases with increasing wavelength and is cut off at wavelengths exceeding $\sim R_{\text {eff }} \theta_{\text {ap }}^{2} \approx b^{2} / R_{0}$. However, using a mirror with no hole is not feasible at Aladdin, which requires a long beam lifetime.

Since the flux plots of Fig. 3 are nearly identical to those of a mirror with infinite extent, they also apply for an edge radiation beam line whose circular entrance aperture has a
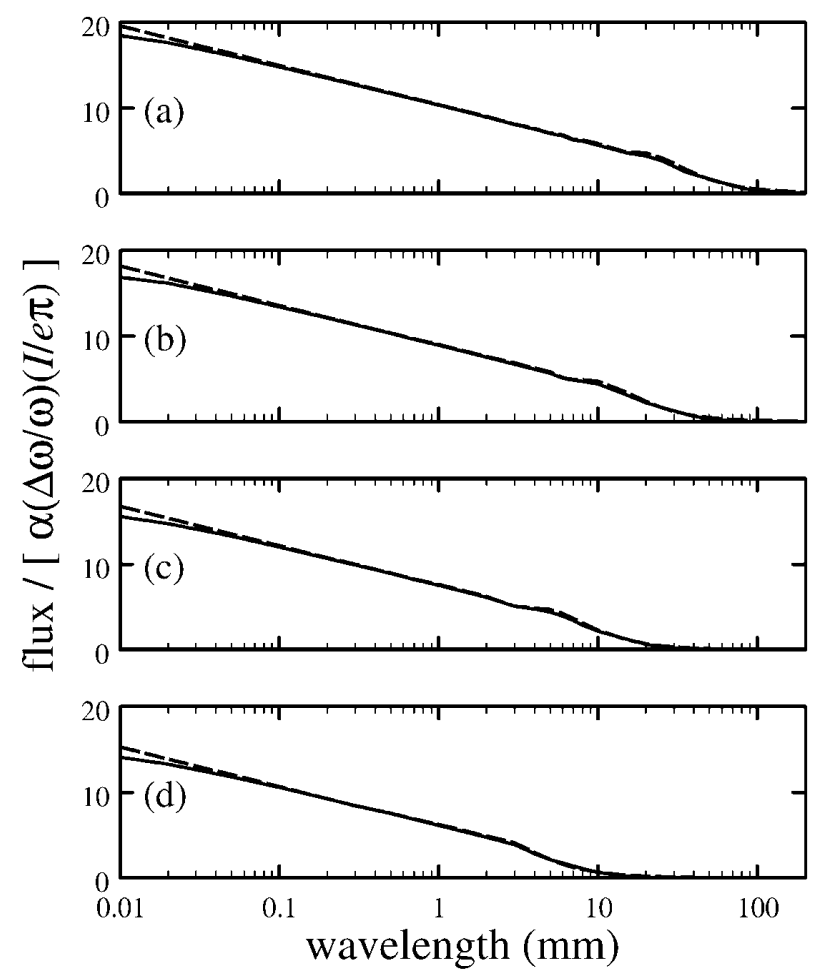

FIG. 3. Extracted flux versus wavelength from a mirror with no hole, for different values of $R_{0}$, the distance from the axis to the extraction window. Solid line: computed flux. Dashed line: flux given by Eq. (18) for a mirror of infinite extent. (a) $R_{0}=32 \mathrm{~mm}$, (b) $R_{0}=64 \mathrm{~mm}$, (c) $R_{0}=128 \mathrm{~mm}$, and (d) $R_{0}=256 \mathrm{~mm}$. radius of $32 \mathrm{~mm}$, located $32,64,128$, or $256 \mathrm{~mm}$ downstream of a 3-m short straight section. However, the closest feasible entrance-aperture location is $1 \mathrm{~m}$ downstream of an Aladdin short straight section, in which case we expect that unreflected radiation is cut off by the vacuum chamber for $\lambda>500 \mu \mathrm{m}$ [9], while reflected radiation is shielded for $\lambda>3 \mathrm{~mm} \mathrm{[22].}$

To allow passage of the beam without reducing its lifetime, measurements indicate that a mirror in a straight section must have a hole or slot with half-height exceeding $9.5 \mathrm{~mm}$; we consider a hole with radius $a=9.5 \mathrm{~mm}$. Since the hole subtends a half-angle of $4.96 / \gamma$ with respect to the upstream end of the straight section, the mirror extracts edge radiation without intercepting the velocity field of the straight section.

Figure 4 shows the radiation from an electron for a window mounted in a standard flange $\left(R_{0}=64 \mathrm{~mm}\right)$, computed from Eq. (14) for $a=9.5 \mathrm{~mm}$. Nearly the same results are obtained from Eq. (14) when $E_{\text {Coul }}$ and $E_{v_{\text {entrance }}}$ are neglected, confirming that the extracted radiation is mostly edge radiation. For wavelengths less than $1 \mathrm{~mm}$, the radiation is a specular reflection with slight diffraction effects; nearly all of the extracted flux passes through the window. At longer wavelengths, where the Fresnel number $b^{2} / \lambda R_{0}$ does not greatly exceed 1 , diffraction becomes important, and the flux through the window is less than the

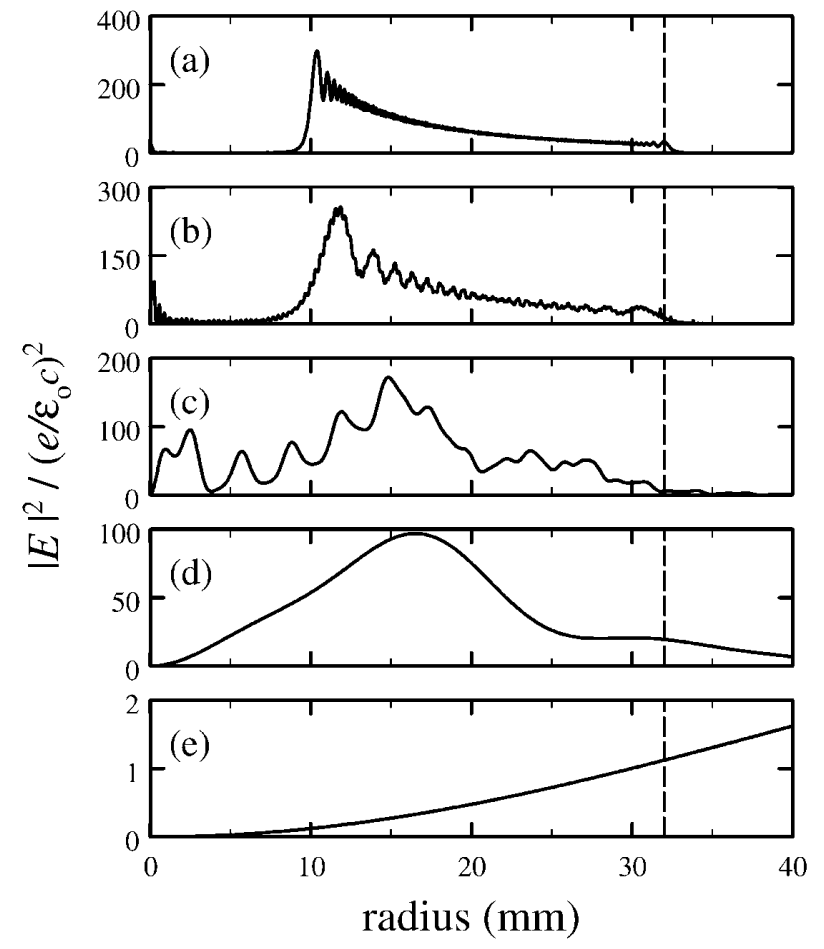

FIG. 4. Radiation distribution at the extraction window from a mirror with a hole radius of $9.5 \mathrm{~mm}$ when the window is located $64 \mathrm{~mm}$ from the straight section axis. The dashed line shows the 32-mm radius of the window. (a) $\lambda=10 \mu \mathrm{m}$, (b) $\lambda=$ $100 \mu \mathrm{m}$, (c) $\lambda=1 \mathrm{~mm}$, (d) $\lambda=10 \mathrm{~mm}$, and (e) $\lambda=100 \mathrm{~mm}$. 
flux incident upon the mirror. As in Fig. 2, it is evident that the finite mirror radius has little affect on the flux through the window, since the mirror is as large as the window.

The computed incoherent flux through the window and the approximate flux given by Eq. (19) are shown in Fig. 5. Equation (19) is in good agreement at wavelengths well below the cutoff, and approximately gives the cutoff wavelength. However, Eq. (19) overestimates the extracted flux near the cutoff wavelength of $\sim 2 \pi a \theta_{\text {ap }} \approx 2 b^{2} / R_{0}$.

The cutoff wavelengths are comparable with and without a hole in the mirror. A mirror without a hole has the advantage of extracting a larger flux at shorter wavelengths. A mirror with a hole has the advantage of minimal perturbation to the beam (so that it is feasible at Aladdin) and gives a flux that is nearly independent of wavelength over a large wavelength range.

For a window flush with the vacuum chamber wall $\left(R_{0}=32 \mathrm{~mm}\right)$, a mirror with a hole gives an incoherent flux of $2.36 \alpha(\Delta \omega / \omega)(I / e \pi)$ for $1 \mu \mathrm{m}<\lambda<60 \mathrm{~mm}$, equaling $6.8 \times 10^{12}$ photons $/ \mathrm{s}-0.1 \%$ bandwidth for a ring current of $200 \mathrm{~mA}$. The cutoff wavelength is decreased if the window is placed farther from the straight section axis, so that a standard flange geometry with $R_{0}=64 \mathrm{~mm}$ has a wavelength cutoff of $\sim 30 \mathrm{~mm}$. Since the incoherent flux is nearly independent of wavelength for $1 \mu \mathrm{m}<\lambda<$ $30-60 \mathrm{~mm}$, the coherent edge radiation may be a useful

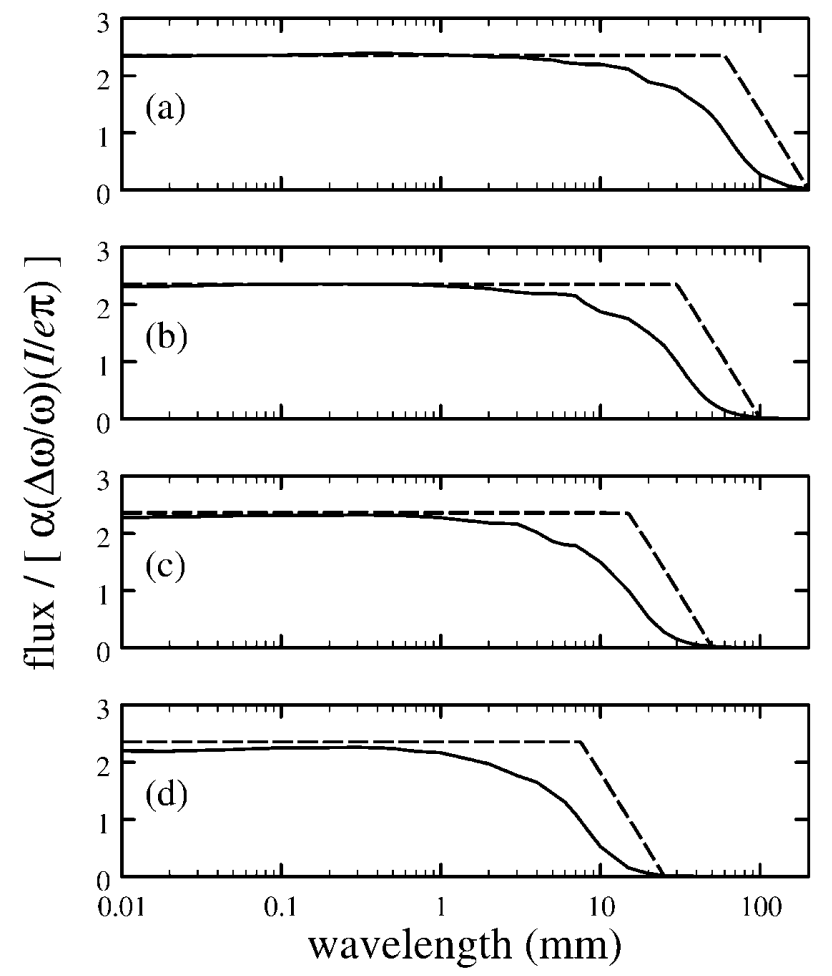

FIG. 5. Extracted flux versus wavelength from a mirror with a hole radius of $9.5 \mathrm{~mm}$, for different values of $R_{0}$, the distance from the axis to the extraction window. Solid line: computed flux. Dashed line: approximate flux from Eq. (19). (a) $R_{0}=32 \mathrm{~mm}$, (b) $R_{0}=64 \mathrm{~mm}$, (c) $R_{0}=128 \mathrm{~mm}$, and (d) $R_{0}=256 \mathrm{~mm}$. diagnostic of the bunch length. The edge radiation at short wavelengths may be focused to determine the transverse beam dimensions at the bending magnet edge $[15,23]$. In addition, the edge radiation may be used as a broadband infrared and microwave source [23].

\section{CONCLUSION}

Using Kirchoff scalar diffraction theory, we modeled the extraction of edge radiation by a tilted mirror with a hole within a straight section of Aladdin. For wavelengths less than $1 \mathrm{~mm}$, the radiation extracted through a nearby window is approximately a specular reflection of the edge radiation incident upon the mirror. At longer wavelengths, diffraction becomes important. When radiation is extracted through a window close to the mirror, the incoherent flux for a ring current of $200 \mathrm{~mA}$ is $6.8 \times 10^{12}$ photons $/ \mathrm{s}-0.1 \%$ bandwidth for wavelengths of $1 \mu \mathrm{m}-60 \mathrm{~mm}$. The edge radiation may be useful as a diagnostic of the bunch dimensions or as a broadband source.

\section{ACKNOWLEDGMENTS}

The author appreciates valuable discussions with J. J. Bisognano, O. V. Chubar, M. V. Fisher, M. A. Green, T. E. May, R. Reininger, and W.S. Trzeciak. This work was supported by NSF Grant No. DMR-0084402.

[1] Yu. N. Dnestrovskii and D. P. Kostomarov, Sov. Phys. Dokl. 4, 132 (1959).

[2] Y. Shibata, S. Hasebe, K. Ishi, T. Takahashi, T. Ohsaka, M. Ikezawa, T. Nakazato, M. Oyamada, S. Urasawa, T. Yamakawa, and Y. Kondo, Phys. Rev. E 52, 6787 (1995).

[3] A.P. Potylitsyn, Nucl. Instrum. Methods Phys. Res., Sect. B 145, 169 (1998).

[4] M. Castellano, V. A. Verzilov, L. Catani, A. Cianchi, G. Orlandi, and M. Geitz, Phys. Rev. E 63, 056501 (2001).

[5] R. A. Bosch, Rev. Sci. Instrum. (to be published).

[6] Y.-L. Mathis, P. Roy, B. Tremblay, A. Nucara, S. Lupi, P. Calvani, and A. Gerschel, Phys. Rev. Lett. 80, 1220 (1998).

[7] P. Roy, Y.-L. Mathis, A. Paolone, P. Giura, A. Nucara, S. Lupi, P. Calvani, and A. Gerschel, Nuovo Cimento Soc. Ital. Fis. 20D, 415 (1998).

[8] P. Roy, M. Cestelli Guidi, A. Nucara, O. Marcouille, P. Calvani, P. Giura, A. Paolone, Y.-L. Mathis, and A. Gerschel, Phys. Rev. Lett. 84, 483 (2000).

[9] R. A. Bosch, Nucl. Instrum. Methods Phys. Res., Sect. A 454, 497 (2000).

[10] J.D. Jackson, Classical Electrodynamics (Wiley, New York, 1975), 2nd ed.

[11] I. S. Gradshteyn and I. M. Ryzhik, Table of Integrals, Series, and Products (Academic Press, San Diego, 1994), 5th ed., p. 517, Eq. 3.915.2.

[12] I. S. Gradshteyn and I. M. Ryzhik, Table of Integrals, Series, and Products (Ref. [11]), p. 697, Eq. 6.521.2.

[13] I.S. Gradshteyn and I. M. Ryzhik, Table of Integrals, Series, and Products (Ref. [11]), p. 759, Eqs. 6.686.5 and 6.686.6. 
[14] R. A. Bosch and O. V. Chubar, in Synchrotron Radiation Instrumentation, edited by E. Fontes, AIP Conf. Proc. No. 417 (AIP, New York, 1997), p. 35.

[15] R. A. Bosch, Nucl. Instrum. Methods Phys. Res., Sect. A 431, 320 (1999).

[16] I.S. Gradshteyn and I. M. Ryzhik, Table of Integrals, Series, and Products (Ref. [11]), p. 697, Eqs. 6.521.2 and 6.521.4; p. 979, Eq. 8.473.1; p. 982, Eq. 8.486.17.

[17] X. Artru, M. Castellano, L. Catani, R. Chehab, D. Giove, K. Honkavaara, P. Patteri, M. Taurigna-Quere, A. Variola, and L. Wartski, Nucl. Instrum. Methods Phys. Res., Sect. A 410, 148 (1998).

[18] O. V. Chubar and N. V. Smolyakov, in Proceedings of the 1993 Particle Accelerator Conference, Washington, DC
(IEEE, Piscataway, NJ, 1993), p. 1626.

[19] R. A. Bosch, in Proceedings of the 1999 Particle Accelerator Conference, New York (IEEE, Piscataway, NJ, 1999), p. 2397.

[20] U. Schade, A. Röseler, E. H. Korte, M. Scheer, and W. B. Peatman, Nucl. Instrum. Methods Phys. Res., Sect. A 455, 476 (2000).

[21] M. A. Green, D. L. Huber, E. M. Rowe, and B. Tonner, Rev. Sci. Instrum. 63, 1582 (1992).

[22] R. A. Bosch, Nucl. Instrum. Methods Phys. Res, Sect. A (to be published).

[23] T. E. May, R. A. Bosch, and R. L. Julian, in Proceedings of the 1999 Particle Accelerator Conference, New York (Ref. [19]), p. 2394. 